\title{
Integrating semantic NLP and logic reasoning into a unified system for fully-automated code checking \\ Jiansong Zhang ${ }^{1}$; and Nora M. El-Gohary ${ }^{2}$
}

\begin{abstract}
Existing automated compliance checking (ACC) systems are limited in their automation; they rely on the use of hard-coded, proprietary rules for representing regulatory requirements, which requires major manual effort in extracting regulatory information from textual regulatory documents and coding these information into a rule format.To address this limitation, this paper proposesa new unified ACCsystem that integrates: (1) semantic natural language processing techniques and EXPRESS data based techniques to automatically extract and transform both regulatory information (in regulatory documents) and design information[in building information models (BIMs)]for automated compliance reasoning, and (2) semantic logic-based information representation so that the reasoning could be fully automated. To test the proposed system, a BIM test case was checked for compliance with Chapter 19 of the International Building Code 2009. Comparing to a manually-developed gold standard, $98.7 \%$ recall and $87.6 \%$ precision in noncompliance detection were achieved.
\end{abstract}

Keywords: Automated code checking; Automated information extraction; Automated reasoning;Building information modeling (BIM); Natural language processing; Logic; Semantic systems; Automated construction management systems.

\footnotetext{
${ }^{1}$ Assistant Professor, Dept. of Civil and Construction Engineering, Western Michigan University, $1903 \mathrm{~W}$ MichiganAve, Kalamazoo, MI 49008.

${ }^{2}$ Assistant Professor, Dept. of Civil and Environmental Engineering, Univ. of Illinois at UrbanaChampaign, 205 N. Mathews Ave., Urbana, IL 61801 (corresponding author). Email:gohary@illinois.edu; Tel: +1-217-333-6620; Fax: +1-217- 265-8039.
} 


\section{Introduction}

21 The manual process of regulatory compliance checking is time-consuming, costly, and error-

22 prone (Boken and Callaghan 2009). In the U.S., each building compliance review cycle usually takes several weeks (State of New Jersey 2014; City of Philadelphia 2015), and a construction project may be subject to multiple cycles of plan reviews due to design changes. At the city level, millions of dollars are spent on manual building compliance checking each year (Department of set of plans (Fiatech Regulatory Streamlining Committee 2012).

In comparison to manual compliance checking, automated compliance checking (ACC) of construction projects is expected to reduce the time, cost, and errors of the compliance checking process (Eastman et al. 2009, Tan et al. 2010; Nguyen and Kim 2011; Kasim et al. 2013; Zhang and El-Gohary 2013).However, the state-of-the-art ACC systems cannot achieve full automation several ACC efforts hard-coded rules for specific subdomains such as building evacuation (Choi et al. 2014), fall protection (Zhang et al. 2013), construction quality (Zhong et al. 2012), building safety design (Qi et al. 2011), building envelope performance (Tan et al. 2010), and accessibility 
43 (Lau and Law 2004). Such hard-coded rules could be very effective in reasoning about

44 compliance with a specific set of requirements and specific regulatory sections in a certain period

45 of time, but such rigid and static representation requires great effort in (1) adaptation to different

46 regulatory codes/sections, and (2) maintenance/update across different time periods and in

47 response to code revisions/updates. The use of hard-coded rules, thus, becomes effort-intensive

48 and time-consuming because of the large number of codes and regulations and their frequent

49 revisions/updates (Delis and Delis 1995; Dimyadi and Amor 2013).

50 In view of that, a number of researchers explored the development of generalized representations

51 for the formalization of regulatory requirements, with the aim to facilitate soft coding of rules for

52 supporting ACC. For example,Pauwels et al. (2011) proposed a semantic rule checking

53 environment, in which Notation 3 (N3) Logic is used to represent requirement rules.Hjelseth and

54 Nisbet (2011) proposed the Requirement, Applies, Select, and Exception (RASE) method to

55 represent regulatory requirements.Yurchyshyna et al. (2010; 2008) developed a conformity-

56 checking ontology that represents regulatory information, building-related knowledge, and

57 expert knowledge on checking procedures, with a representation of regulatory requirements in

58 the form of SPARQL Protocol and RDF Query Language (SPARQL) queries. Beach et al. (2013;

59 2015) extended the RASE methodfor a more powerful regulatory information representation at

60 both "the block level (i.e., paragraph level) and inline (i.e., individual words or groups of

61 words)", which can be converted to Semantic Web Rule Language (SWRL) for

62 reasoning.And,Dimyadi et al. (2014) represented regulatory requirements using the Drools Rule

63 Language (DRL).

64 These efforts have undoubtedly contributed to the improvement of flexibility and reusability of

65 regulatory representations for supporting ACC. However, they are still limited in terms of 
automated regulatory information extraction and transformation; the state of the art in ACC still

67 requires major manual efforts in extracting regulatory information from textual regulatory documents and transforming/encoding these information into a computer-processable rule format.

69 For example, inPauwels et al. (2011),Hjelseth and Nisbet (2011), Yurchyshyna et al. (2010; 2008), Beach et al. (2013; 2015), and Dimyadi et al. (2014), the extraction of regulatory

71 information and their encoding into N3Logic, the RASE representation, the SPARQL queries,

72 the extended RASE representation, and the DRL rules, respectively, are still manually conducted.

73 To facilitate the regulatory information extraction and conversion, the SMARTcodes project led 74 by the International Code Council (ICC) developed tools to help ICC staff and building code 75 officials mark-up the ICC codes with provided tags under a predefinedSMARTcodes schema. 76 The marked codes, then, can be automatically transformed into a "requirements model", which 77 leverages the IfcConstraintentities within an Industry Foundation Classes (IFC) model and 78 therefore is essentially an IFC constraint model (AEC3 2012). As the process suggests, the 79 SMARTcodes project still requires manual rule extraction and encoding efforts in the form of 80 marking-up tasks.

81 To address these gaps of knowledge, this paper proposes a new fully-automated ACC system 82 [the authors call it semantic natural language processing(NLP)-based automated compliance 83 checking (SNACC) system] that integrates three types of algorithms in one unified 84 computational platform: (1) semantic NLP) algorithms to automatically extract the regulatory 85 information from regulatory documents(e.g., building codes) and transforms the extracted 86 regulatory information into logic rules, (2) semantic EXPRESS data processing algorithms to 87 automatically extract the design information from building information models and transform the 88 extracted design information into logic facts, and (3) semantic-based logic reasoning algorithms 
to automatically reasonabout the compliance of the logic facts with the logic rules. The automated analyses are facilitated by information representationsthat are semantic, logic-based,

91 and generalized and flexible. This paper presents the integration of the proposed algorithms in a

92 unified ACC system and discusses the experimental results of testing the proposed unified 93 system using a test case.

\section{Proposed approach to full automation in automated compliance checking}

95 This paper proposes a fully-automated approach to ACC in construction. The approach relies on 96 the use of a set of computational techniques in an integrated manner, in one unified system. The 97 techniques include NLP, EXPRESS data processing, and logic reasoning, which are collectively 98 used for automated information processing (both design information and regulatory information) 99 and automated compliance reasoning. The automated processes are facilitated by semantic, 100 logic-based representations that are generalized and flexible.

$101 \quad 2.1 \quad$ Information representation

102 The choice of information representation has strong implications on information processing and 103 is of vital importance in facilitating automated processes. In ACC applications, specifically,there 104 is a need for a "standard, generalized approach for formally representing building regulations in 105 a digital format that would facilitate a variety of forms of reasoning about those codes in 106 combination with digital building information models” (Garrett et al. 2014), including automated 107 information extraction and information transformation to support complete automation of ACC. 108 The proposed representation is semantic and logic-based, in a way which is generalized and 109 flexible.

\subsubsection{Semantic representation}


111 The representation is semantic; it uses semantic information elements and a domain ontology.

112 Semantic information elementsrepresent the elementsof a regulatory requirement, including 113 “subject,” “compliance checking attribute,” “deontic operator indicator,” “quantitative 114 relation,”“comparative relation,” “quantity value,” “quantity unit,” “quantity reference,” 115 “restriction,” or “exception.”A building ontology is asemantic model for representing building 116 domain knowledge in the form of concept hierarchies, relationships between concepts, and 117 axioms. The semantic representation facilitates deep information processing (i.e., full-sentence 118 analysis towards capturing the entire meaning of a sentence, as opposed to shallow processing 119 that extracts partial information from a sentence). The semantic representation is also utilized to 120 leverage domain knowledge in the reasoning process, in order to handle the complex relations 121 involved in compliance reasoning and enable deep reasoning. This is important because the 122 relations in regulatory provisions could be very complex. For example, Fig. 1 shows the many 123 relations involved in one single regulatory provision in IBC 2006, leading to a very complex 124 regulatory provision. The semantic representation also facilitates human understandability and 125 interpretability of the formal representation, which is essential to facilitate usability and allow for 126 human testing and verification of the information representation and the reasoning results. 
The minimum required net free ventilating area shall be 1/300 of the area of the space ventilated, provided a vapor retarder having a transmission rate not exceeding 1 perm in accordance with ASTM E 96 is installed on the warm side of the attic insulation and provided 50 percent of the required ventilating area provided by ventilators located in the upper portion of the space to be ventilated at least 3 feet above eave or cornice vents, with the balance of the required ventilation provided by eave or cornice vents.
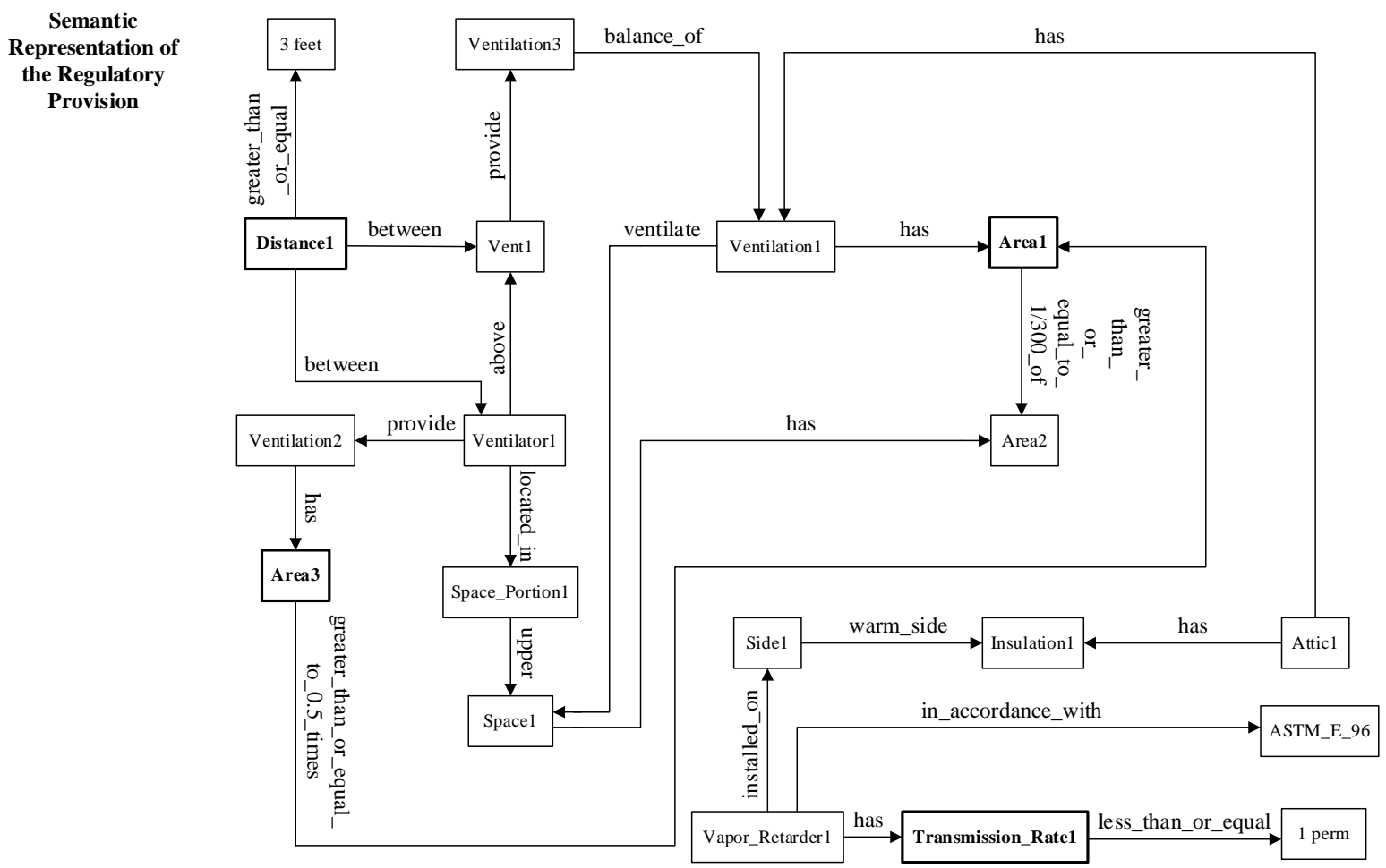

Fig.1.An example to illustrate the complexity of relations in provisions.

\subsubsection{Logic representation}

The representation is logic-based: regulatory information are represented as logic rules, while design information are represented as logic facts. A logic-based representation was selected to take advantage of the well-matured logic-based reasoning techniques. Logic-based reasoning is well-suited for ACC problems because (Zhang and El-Gohary 2016b): (1) The binary nature (satisfy or fail to satisfy) of logic fits the binary nature (compliance or noncompliance) of ACC; (2) Formally-defined logics have sufficient expressiveness to represent concepts and relations involved in ACC; (3) Once the information is properly represented in a logic format, the reasoning can be conducted in a fully-automated way; and (4) Automated reasoning techniques are available in ready-to-use logic reasoners. 
139 Among the existing types of logic, FOL is the foundation of almost all work in rule 140 representation for ACC because of its expressivity; these efforts used a variety of logic 141 implementations/languages, but they all built on some restricted form of FOL. For example, 142 Pauwels and Zhang (2015) reviewed a good number of semantic rule checking applications 143 among which two main types of logic were used: N3Logic (e.g., Dimyadi et al. 2015) and SWRL 144 (e.g., Baumgärtel et al. 2015). Both N3Logic and SWRL were created to go beyond the 145 monotonic negation limitation of FOL. N3Logic was created to avoid the paradox traps problem 146 of FOL by not using the general first-order negation but rather relying on customarily-made 147 negated forms of functions to achieve nonmonotonic negation (Berners-Lee 2005). SWRL is 148 essentially combining theDatalogRule Markup Language (RuleML) with the Web Ontology 149 Language (OWL), where Datalog is a restricted subset of FOL using function-free Horn Clauses 150 (HCs) (Horrocks et al. 2004). A HC is a restricted form of FOL thatis most efficient in inference 151 making (Saint-Dizier 1994). Both N3Logic and SWRL were used because of their compatibility 152 with OWL ontologies, which are the core of semantic rule checking approaches. More 153 importantly, logic such as N3Logic and SWRL need to be used to support if-then rule 154 representation for rule checking when OWL ontologies are used, because OWL is based on 155 description logic (DL). A set of rules (if-then statements) is necessary to allow for rule checking 156 (Pauwels and Zhang 2015), and DL does not allow for the representation of if-then rules. In 157 addition to N3Logic and SWRL, Solihin and Eastman (2015) took a knowledge representation 158 approach for representing requirement rules using conceptual graphs, which also has a semantic 159 foundation in FOL and has one-to-one mapping to FOL rules.

160 FOL was selected, in this paper, to support ACC not only because of its expressivity but also 161 because of its ability to represent English sentences. “Afirst-ordersentence $\varphi c a n$ oftenbe 
162 translated into an English sentence which is guaranteed to be true if and only if pis true in I" (i.e.,

163 the interpretation) (Hodges 2001). This property makesFOL suitable for representing regulatory

164 information to support automated compliance reasoning, because existing regulatory rules in

165 building codes and regulations are mostly coded in natural language sentences. Although FOL

166 cannot represent all provisions in building codes and regulations (Garrett et al. 2014), among

167 those provisions it can represent, FOL: (1) enables isomorphism: one-to-one mapping between 168 an English regulatory requirement and a logic clause, and (2) as a result, allows for traceability:

169 maintaining traceability is important to identify the sources of logic clauses and, thus, to

170 facilitate human verification and ensure trustworthiness of the logic clauses and the results. The

171 scope of this paper is limitedto quantitative requirements - part of the regulatory requirements

172 that are representable in FOL. The representability of all possible types of regulatory

173 requirements in FOL (i.e., which requirements can be represented in FOL and which not) is an

174 interesting topic that is worth further investigation (Garrett et al. 2014), but is outside of the 175 scope ofthis paper.

\section{$176 \quad$ 2.1.3 Generalized and flexible representation}

177 The representation is generalized and flexible. The generalization and flexibility are achieved 178 through generalized regulatory compliance checking concepts and flexible semantic information 179 elements. Generalized regulatory compliance checking concepts (e.g., "subject” and “compliance 180 checking attribute”) are used, which allows for representing regulatory provisions of any 181 type/topic (e.g., building envelope performance, facility accessibility). Flexible information 182 elements (e.g., "subject restriction," as discussed in the following sections) are used, which 183 allows for representing all information (i.e., all concepts and relations) in a regulatory provision 184 regardless of the length and complexity of the provision (sentence). Generalization and 
185 flexibility are important to sustain utility and robustness of the proposed system across different 186 types of regulatory documents and different types of provisions.

\subsection{Computational techniques}

\subsubsection{Deepnatural language processing techniques}

189 It is an important impact conceived by many researchers who work on computable regulatory 190 rule representations (e.g., RASE, SMARTcodes) that the use oftheir representations may guide 191 the future drafting of codes and regulations (e.g., through the use of built-in annotations), so that 192 the automated extraction and transformation of regulatory information into computable rules 193 would be easily addressed. The authors also share that aspiration. But, at the same time, the 194 authors foresee that long-term goal (i.e., changing the way codes and regulations are drafted) as a 195 big challenge, potentially beyond the reach of solely the construction community, because it 196 requires harmonizing a lot of different pursuits and interests from various stakeholders (code 197 drafters, regulators, designers, etc.).Let alone that any developer of a computable regulatory rule 198 representation typically wants their own development to be adopted at a large scale, both 199 geographically and democratically - so which representation becomes a standard or becomes 200 widely adopted is another issue. On the other hand, the authors hold the ground of the status quo 201 that current building codes and regulations are mostly represented in natural language text, and 202 leveragestate-of-the-art NLP techniques to develop new methods towards bridging the 203 automation gap of regulatory information extraction and transformation, under their proposed 204 ACC framework.

205 NLP techniques are used to facilitate text analysis and processing for automatically extracting 206 regulatory information from building codes. NLP is a theoretically-based computerized approach 207 to analyzing, representing, and manipulating natural language text for the purpose of achieving 
208 human-like language processing for a range of tasks or applications (Cherpas 1992). The types of 209 natural language analyses and techniques used highly affect the ability of NLP algorithms to 210 process complex sentences and recognize their full meaning. Full sentence understanding - of

211 both simple and complex sentences - is essential to achieve full automation in analyzing building

212 codes and extracting regulatory information. Deep NLP aims to capture the full meaning of

213 sentences to facilitate full sentence understanding by computers (Zouaq 2011). The proposed 214 approach offers a new way to achieve a deep level of text processing by integrating three types 215 of knowledge in the analysis of sentences: (1) ACC-specific knowledge: knowledge about the 216 elements of a regulatory requirement in building codes, represented in the form of semantic 217 information elements, (2) AEC domain knowledge: knowledge about the building domain, 218 represented in the form of an ontology, and (3) linguistic knowledge: knowledge about the 219 linguistic expressions of requirements in building code provisions, represented in the form of 220 information extraction rules.

\section{$221 \quad$ 2.2.2 EXPRESS data processing techniques}

222 EXPRESS data processing techniques are used for automatically extracting design information 223 from building information models. EXPRESS data processing techniques are suitable for 224 accessing information from IFC-based BIMs because the IFC schema is written in the EXPRESS 225 language. This EXPRESS language-level of processing enables the extraction and further 226 transformation of design information to be aligned with regulatory information.

227 The Java Standard Data Access Interface (JSDAI) was utilized for BIM information extraction, 228 using late binding data access methods. JSDAI is a standard data access interface (SDAI) 229 application programming interface (API) to access information from models written in EXPRESS language -the ISO standard product data modeling language (ISO 2004). JSDAI 
231 provides two types of data access methods: (1) early binding method, which accesses entities and

232 attributes in an EXPRESS model with specialized access methods such as "getCeilingHeight"

233 (i.e., method to get ceiling height of a floor), and (2) late binding method, which accesses entities

234 and attributes in an EXPRESS model with generalized access methods such as

235 "getExplicitAttributes" (i.e., method to get any explicit attribute). Compared to early binding,late

236 binding allows accessing information based on more general metadata.

\section{$237 \quad$ 2.2.3 Logic reasoning and programming}

238 Logic programming is a computational programming paradigm that is based on a Horn Clause 239 (HC)-representation (Portoraro 2011).A program written in a logic programming language is 240 simply a set of logic sentences that represent facts and rules about some domain of interest. 241 Logic programming is declarative in contrast to other non-logical programming languages. For 242 example, in typical procedural programming languages like $\mathrm{C}$ programming language a 243 programmer has to clearly define how to solve the problem step by step, whereas in logic 244 programming a programmer only needs to define how to represent the problem in the form of 245 facts and rules. The solution steps in logic programming are already defined by a built-in 246 reasoner through a set of organized automated reasoning techniques such as search strategies and 247 backtracking.

\section{$248 \quad 2.3 \quad$ System integration}

249 The proposed system offers a novel integration of natural language processing techniques, 250 EXPRESS data processing techniques, and logic reasoning into one unified computational 251 frameworkto allow for full automation in ACC. The integration is facilitated by the choice of (as 252 per Fig. 2): (1) a semantic representation that allows for seamless flow of information from one 253 computational paradigm to another, from one computational module to another, and from one 
254 algorithm to another, (2) a logic representation, as a final representation, which allows to 255 combine partial output from two separate modules (logic rules and logic facts from module 1 and 256 module 2, respectively)into one coherent representation that is ready for reasoning, (3) a modular 257 system architecture, whichenables a flexible use of multiple modeling paradigms and multiple 258 programming languages, and (4) an architecture-neutral platform that can interoperate with 259 multiple programming language interfaces.

260

261

262

263

264

265

266

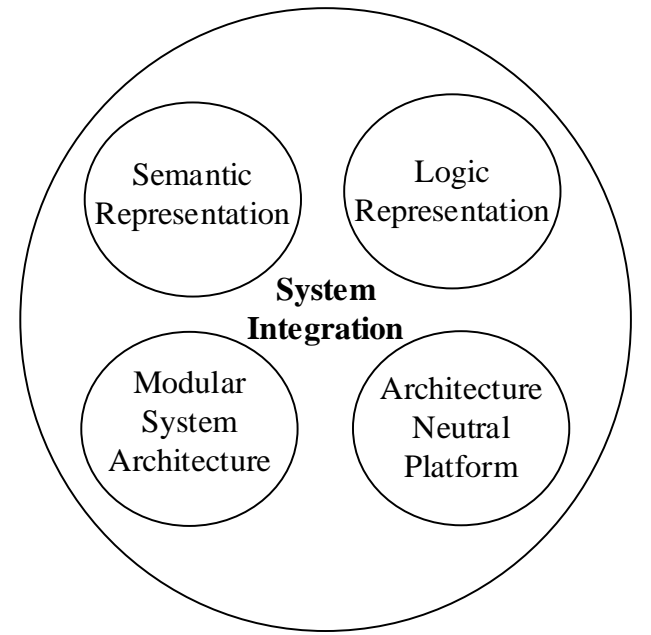

Fig.2.System integration.

\section{System architecture}

This section provides an overview of the system architecture, including an overview of: (1) the system modules and how they are interlinked and integrated, and (2) the implementation of the system modules and how they interact. More details on the individual system modules and implementationsare provided in Sections 4 and 5.

The system architecture is illustrated in Fig. 3. It is composed of three main modules: (1) regulatory information extraction and transformation module, (2) design information extraction and transformation module, and (3) compliance reasoning module. The system architecture is built on top of the Java Platform (Oracle 1999). The General Architecture for Text Engineering (GATE) tools (Cunningham et al. 2012), Python programming language (Python 2.7.3), B- 
272 Prolog logic programming platform and reasoner (Zhou 2012), and JSDAI tools are used in the

274

275

276

277

278

279

280

281

282

283

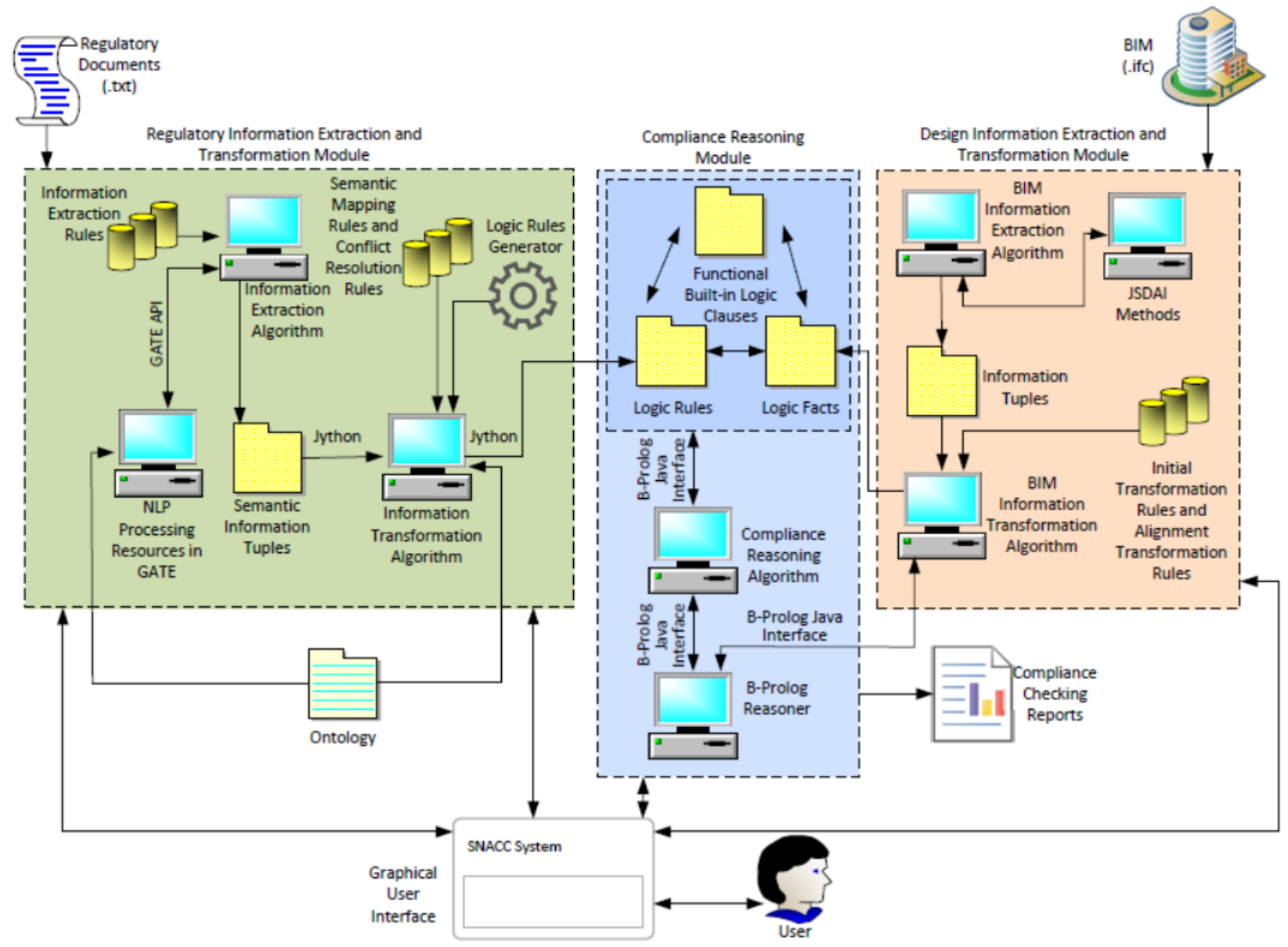

Fig. 3.System architecture of the SNACC system.

The regulatory information extraction and transformation module is composed of the regulatory information extraction algorithm and the regulatory information transformation algorithm. The information extraction algorithm aims to extract the regulatory requirements from a regulatory document into a semantic information tuple representation, where each tuple contains information instances for the semantic information elements (e.g., "subject," "compliance checking attribute”). The algorithm relies on the use of a set of pattern matching-based information extraction rules. A set of syntactic and semantic features are used in the patterns of the information extraction rules. The syntactic features are generated using GATE's Processing 
284 Resources (e.g., tokenizer), while the semantic features are generated from the ontology using

285 GATE’s Processing Resources (e.g., gazetteer). The information extraction algorithm interacts 286 with the Processing Resources using GATE's API in Java. The regulatory information 287 transformation algorithm aims to transformthe extracted instances of the semantic information 288 elements in the information tuples into logic rules. The algorithm relies on the use of a set of 289 pattern matching-based semantic mapping rules and conflict resolution rules, which include a set 290 of syntactic and semantic features in their patterns. The semantic features, here, are the semantic 291 information element features (e.g., the semantic feature "s" stands for "subject"). The 292 information transformation algorithm interacts with the other modules of the SNACC system (in 293 Java) through Jython. The ontology is used to support the regulatory information extraction and 294 transformation processes by facilitating automated interpretability and understandability of 295 regulatory text based on meaning.

296 The design information extraction and transformation module is composed of the BIM 297 information extraction algorithm and the BIM information transformation algorithm. The BIM 298 information extraction algorithm aims to extract the entities and their attributes from a BIM into 299 an information tuple representation. The algorithm relies on the use of a set of entity and 300 attribute extraction rules. The data types of the entities and attributes are extracted from the BIM 301 using late binding data access methods in JSDAI. The BIM information transformation algorithm 302 aims to transform the extracted entities and attributes in the information tuples into logic facts 303 that are aligned with the logic rules. The algorithm relies on the use of initial transformation 304 rules and semantic transformation rules. The initial transformation rules transform the extracted 305 entities and attributes in the information tuples into logic facts. The semantic transformation 306 rules further transform the initially transformed logic facts into more semantic logic facts that are 
307 aligned with the predicates in the logic rules. The initial transformation rules are coded in Java

308 and the semantic transformation rules are coded in B-Prolog rules. To execute thesemantic

309 transformation rules, the information transformation algorithm interacts with B-Prolog's reasoner

310 through B-Prolog’s interface with Java.

311 The compliance reasoning module is composed of the compliance reasoning algorithm,which

312 utilizes B-Prolog's reasoner. The compliance reasoning algorithm aims to reason about the logic

313 rules and the logic facts and generate compliance checking reports. The algorithm controls and

314 supports the reasoning about the rules and facts in B-Prolog's reasoner using a set of functional

315 built-in logic clauses. The compliance reasoning algorithm interacts with B-Prolog's reasoner

316 through B-Prolog's interface with Java. A user interacts with all the three modules through a

317 graphical user interface.

\section{$318 \quad 4 \quad$ System modules}

3194.1 Regulatory information extraction and transformation module

320 The regulatory information extraction and transformation module is composed of four main 321 processes: preprocessing, feature generation, information extraction, and information 322 transformation.

323 Preprocessing prepares the raw natural language text of building codes for further processing.

324 Four NLP techniques are utilized: tokenization, sentence splitting, morphological analysis, and 325 dehyphenation. Tokenization divides the text into tokens (words or terms) to prepare for further 326 unit-based processing of the text. Sentence splitting recognizes the boundaries of the sentences to 327 help distinguish provisions in the building codes. Morphological analysis recognizes the different 328 forms of a word and maps them into the lexical form of that word. This helps in the recognition 
329 of ontology concepts. Dehyphenation removes hyphens that indicate continuation of words

330 between lines to avoid further processing errors caused by those hyphens.

331 Feature generation generates a set of syntactic and semantic features that describe the text. Three

332 NLP techniques are utilized to generate the syntactic features: POS tagging, phrase structure

333 analysis, and gazetteer list analysis. POS tagging tags each word with the POS [lexical and

334 functional categories such as singular or mass noun (NN) and adjective (JJ)] of the word. Phrase

335 structure analysis tags each phrase with the phrasal tag [lexical and functional categories such as

336 noun phrase (NP) and verb phrase (VP)]. A set of application-specific phrase structure grammar

337 (PSG) rules are used to generate phrasal tags. The use of phrasal tags in addition to POS tags

338 reduces the potential number of enumerations in the patterns of the information extraction rules

339 (described in the following step). Gazetteer list analysis identifies each word that belongs to a

340 gazetteer list [a set of names based on any specific commonality possessed by those terms, e.g.,

341 “unit gazetteer list” includes inches and feet among others] and uses that information as a feature.

342 The building ontology is utilized to generate the semantic features, including terms/phrases that

343 match to the concepts and relations in the ontology. Fig. 4 shows a partial view of the ontology

344 that was used. 
347 Information extraction extracts the instances of the semantic information elements (SIEs) from

348 the building code, using a set of 146 information extraction (IE) rules. AnSIE is an ontology 349 concept, an ontology relation, a "deontic operator indicator" (a term indicating an obligation, 350 permission, or prohibition), or a "restriction" (an element that places a constraint on the

351 definition of another semantic information element, where the constraint is expressed in terms of 352 ontology concepts and relations). The ten types of SIEs and their definitions are shown in Table 353 1. Each SIE is either a "simple SIE" or a "complex SIE," and a "rigid SIE" or a "flexible SIE" 354 (Zhang and El-Gohary 2013). A simple SIE is associated with a single concept/relation/indicator 355 whereas a complex SIE is expressed in terms of multiple concepts and relations. The simple SIEs 356 are rigid [has a fixed number (i.e., 1) of concepts/relations], whereas the complex SIEs are 357 flexible [has a varying number (i.e., 0 or more) of concepts/relations]. The IE rules use pattern 358

matching; the rules extract the instances of each SIEbased on text patterns. The patterns consist 
of syntactic and semantic features, which were generated during the feature generation step. For example, an IE rule for extracting the instances of "subject" is shown in Fig. 5. The example IE rules use patterns that consist of semantic features of "building element,” “room,” "space,”and “quantity,” and syntactic features of "modal verb,” “negation,” "base form verb,” “comparative relation,” “cardinal number,” “slash,” and “unit.”The IE rules were developed based on Chapters 12 and 23 of the International Building Code (IBC) 2006 (ICC 2006). The extraction of each semantic information element is separated and arranged in the following sequence because extracting all semantic information elements from a sentence using a single IE rule is not efficient: "quantity value" and "quantity unit/quantity reference" > "subject" > "compliance checking attribute" > “comparative relation” > "quantitative relation" and "deontic operator indicator" > “subject restriction” and "quantity restriction." An example illustrating the extraction is shown in Fig. 5.The text is then tagged with the extracted SIEs for further information transformation.

Information transformation transforms the extracted information into logic rules, using a set of 9 conflict resolution (CR) rules, 297 semantic mapping (SM) rules, and a logic rule generator. The CR rules and SM rules use pattern matching. The patterns consist of three types of information tags: (1) syntactic information tags: syntactic feature tags generated during feature generation, (2) semantic information tags: SIE tags generated during information extraction, and (3) combinatorial information tags: compound information tags that are composed of multiple syntactic and/or semantic information tags. Fig. 6 shows an example of a tagged regulatory requirement. The $\mathrm{CR}$ rules resolve conflicts between the extracted information instances (in the form of four-element tuples) based on the patterns. The SM rules transform the extracted information instances (after conflict resolution) into logic components (i.e., logic predicates and 
logic operators) based on the patterns. For example, ' $n$ ' 'c' 'v' ' $u$ ' is used as a pattern for an SM “quantity unit.” Fig. 7 shows an example of an SM rule.

385 Table 1.

386 Semantic information elements (Zhang and El-Gohary 2016b; 2013).

\begin{tabular}{|c|c|c|}
\hline $\begin{array}{c}\text { Semantic information } \\
\text { element }\end{array}$ & Definition & Type \\
\hline Subject & $\begin{array}{l}\text { An ontology concept that describes a "thing" (e.g., building object, } \\
\text { space) that is subject to a particular regulation or norm. }\end{array}$ & Simple and rigid SIE \\
\hline $\begin{array}{c}\text { Compliance checking } \\
\text { attribute }\end{array}$ & $\begin{array}{l}\text { An ontology concept that describes a specific characteristic of a } \\
\text { "subject" by which its compliance is assessed. }\end{array}$ & Simple and rigid SIE \\
\hline $\begin{array}{l}\text { Deontic operator } \\
\text { indicator }\end{array}$ & $\begin{array}{l}\text { A term or phrase that indicates the deontic type of the requirement } \\
\text { (i.e., whether it is an obligation, permission, or prohibition). }\end{array}$ & Simple and rigid SIE \\
\hline Quantitative relation & $\begin{array}{l}\text { A term or phrase that defines the type of relation for the quantity } \\
\text { (e.g., "increase" is a quantitative relation). }\end{array}$ & Simple and rigid SIE \\
\hline Comparative relation & $\begin{array}{l}\text { An ontology relation that is commonly used for comparing } \\
\text { quantitative values (i.e., comparing an existing value to a required } \\
\text { minimum, maximum, or exact value), including "greater than or } \\
\text { equal to," "greater than," "less than or equal to," "less than,” and } \\
\text { "equal to." }\end{array}$ & Simple and rigid SIE \\
\hline Quantity value & $\begin{array}{l}\text { A data value (or a range of values) that defines the quantified } \\
\text { requirement. }\end{array}$ & Simple and rigid SIE \\
\hline Quantity unit & The unit of measure for a "quantity value.” & Simple and rigid SIE \\
\hline Quantity reference & $\begin{array}{l}\text { A term or phrase that refers to another quantity (which includes a } \\
\text { value and a unit). }\end{array}$ & Simple and rigid SIE \\
\hline Subject restriction & $\begin{array}{l}\text { A term, phrase, or clause (which is composed of one or more } \\
\text { concepts and/or relations) that places a constraint on the "subject." }\end{array}$ & $\begin{array}{l}\text { Complex and flexible } \\
\text { SIE }\end{array}$ \\
\hline Quantity restriction & $\begin{array}{l}\text { A term, phrase, or clause (which is composed of one or more } \\
\text { concepts and/or relations) that places a constraint on the } \\
\text { "quantity." }\end{array}$ & $\begin{array}{l}\text { Complex and flexible } \\
\text { SIE }\end{array}$ \\
\hline
\end{tabular}




\section{Original Text:}

The thickness of concrete floor slabs supported directly on the ground shall not be less than $31 / 2$ inches.

\section{Text with Features:}

The thickness (ontology concept "quantity") of concrete floor slabs (ontology concept "building element") supported directly on the ground shall (POS tag "MD" for modal verb) not (gazetteer list "Negation") be (POS tag "VB" for base form verb) less than (gazetteer list "Comparative relation") 31(POS tag "CD" for cardinal number)/(POS tag "Slash" for a slash)2(POS tag "CD") inches (gazetteer list "Unit").

\section{IE Rules:}

If "MD + Negation + VB + Comparative Relation" is matched, extract the text matched with "Negation" and the text matched with "Comparative relation" together as an instance for "comparative relation."

If ontology concept "building element" or "space" or "room" is matched, extract the matched text as an instance for "subject."

If ontology concept "quantity" is matched, extract the matched text as an instance for "compliance checking attribute."

If " $\mathrm{CD}+$ Slash + CD + Unit" is matched, extract the text matched with " $\mathrm{CD}+\mathrm{Slash}+\mathrm{CD}$ " as an instance of "quantity value," extract the text matched with "Unit" as an instance of "quantity unit."

Extracted Instances:

"thickness" as a "compliance checking attribute"

"concrete floor slab" as a "subject"

"not less than" as a "comparative relation"

" $31 / 2$ " as a "quantity value"

"inches" as a "quantity unit"

1. For simplicity only features related to the IE rules below are displayed.

Fig. 5.Sample information extraction rules and extracted instances. 


\section{Original Text}

The thickness of exterior basement walls and foundation walls shall be not less than $71 / 2$ inches.

\section{Information Tags}

- Semantic informati on tags: ' $s$ ' for subject, ' $a$ ' for compliance checking attribute, ' $c$ ' for comparative relation, 'v' for quantity value, 'u' for quantity unit;

- Syntactic informati on tags: 'CC' for conjunctive term, 'CD' for cardinal number, 'IN' for preposition, 'JJ' for adjective, 'MD' for modal verb, 'TO' for literal "to," 'VB' for base form verb, 'VBN' for past participle verb;

- Combinatorial information tags: 'dpvr' for directional passive Verbal relation, which is the combination of "past participle verb" (POS tag "VBN") and "preposition" (POS tag "IN").

Information Tuples Using Three Types of Information Tags ${ }^{1}$

[('thickness', 4, 9, 'a'), ('thickness', 4, 9, 'cr'), ('of', 14, 2, 'OF'), ('of', 14, 2, 'IN'), ('exterior basement walls', 17, 23, 's'), ('exterior', 17, 8, 'cr'), ('basement', 26, 8, 'cr'), ('walls', 35, 5, 'cr'), ('and', 41, 3, 'CC'), ('foundation walls', 45, 16, 's'), ('foundation', 45, 10, 'cr'), ('walls', 56, 5, 'cr'), ('shall', 62, 5, 'MD'), ('be', 68, 2, 'VB'), ('not', 71, 3, 'n'),

(less than', 75, 9, 'c'), ('less', 75, 4, 'JJR'), ('than', 80, 4, 'IN'), ('71/2', 85, 4, 'v'), ('71/2', 85, 4, 'CD'), ('inches', 90, 6 , 'u'), ('inches', 90, 6, 'cr')]

\section{Information Tuples with Conflict Resolution Rules Applied ${ }^{1}$}

[('thickness', 4, 9, 'a'), ('of', 14, 2, 'OF'), ('exterior basement walls', 17, 23, 's'), ('and', 41, 3, 'CC'), ('foundation walls', 45, 16, 's'), ('shall', 62, 5, 'MD'), ('be', 68, 2, 'VB'), ('not', 71, 3, 'n'), ('less_than', 75, 9, 'c'), ('71/2', 85, 4, 'v'), ('inches', 90, 6, 'u')]

\section{Logic Components after Applying Semantic Mapping Rules ${ }^{2}$}

thickness(Thickness),(exterior basement_wall(Exterior_basement_wall),foundation_wall(Exterior_basement_wall )), has(Exterior_basement_wall, Thickness),not less_than(Thickness, quantity(71/2,inches))

\section{Logic Rules Generated by Logic Rule Generator (Partial) ${ }^{2}$}

Primary Logic Clause

compliance_thickness_of_Exterior_basement_wall81(Exterior_basement_wall):-

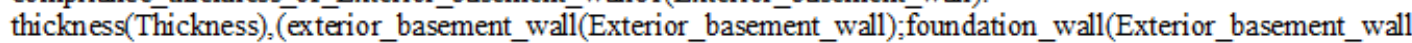
)),has(Exterior_basement_wall, Thickness),not less_than(Thickness, quantity $(71 / 2$,inches $)$ ).

Activation Condition Logic Clause

...thickness(Thickness),(exterior_basement_wall(X);foundation_wall(X)),has(X,Thickness)-> check_thickness_of_Exterior_basement_wall181(X);true,...

\section{Compliance Checking Consequence Logic Clause}

check_thickness_of_Exterior_basement_wall81(X):-(compliance_thickness_of_Exterior_basement_wall81(X)-> writeln((X,is, compliant, with, section, 1909-6-1, rule81)); writeln((X,is, noncompliant, with, section, 1909-61 , thickness, should, be, not,less_than, $71 / 2$, inches,rule82))).

1. Each tuple includes four elements: the information instance, its location (the starting point in the sentence), its length (in number of letters), and its information tag.

2. In this logic syntax, comma represents conjunction, semicolon represents disjunction, "not" represents negation, ":-" represents implication, predicate takes the form of pred $(\arg 1, \arg 2, \ldots)$, rule takes the form of predh $(\arg 1, \arg 2, \ldots):-$ $\operatorname{pred1}(\arg 1, \arg 2, \ldots)$, pred2 $(\arg 1, \arg 2, \ldots) \ldots, \operatorname{predn}(\arg 1, \arg 2, \ldots)$.

Fig. 6.An Example to illustrate regulatory information transformation.

SM Rule Pattern

'subject' (s) 'negation' 'comparative relation’ (cr) ‘value’ (v) ‘unit’ (u)

\section{Logic Clause}

s(S),not cr(S,quantity $(v, u))$

Fig.7. An example of a semantic mapping rule. 
394 The logic rule generator generates three types of logic rules based on the logic components:

395 primary logic clauses, activation condition logic clauses, and compliance checking consequence

396 logic clauses. A primary logic clause is the main representation of a requirement; the premise of

397 the rule represents the conditions of a requirement and the conclusion of the rule represents the

398 consequent result (i.e., the compliance with the requirement). For example, in the primary logic

399 clause in Fig. 6, the logic components to the right of “:-” represent the conditions of the wall

400 thickness requirement (for exterior basement walls and foundation walls) and the logic

401 components to the left of “:-" represent the conclusion of that requirement. An activation

402 condition logic clause represents the conditions that activate the checking of a requirement,

403 which are the existence of the corresponding information in the BIM (e.g., the existence of

404 exterior basement wall or foundation wall and thickness information for the example in Fig. 6).

405 Activation conditions are used to help prevent missing information from leading to false

406 positives because missing information would lead to failure in activation. A compliance

407 checking consequence logic clause represents the consequences of the compliance checking

408 result (compliance or noncompliance). For example, if the result is noncompliant, a corrective

409 suggestion is provided (e.g., "thickness should be not less than 71/2inches,” as per Fig. 6).

\subsection{Design information extraction and transformation module}

411 The design information extraction and transformation module is composed of two main 412 processes: BIM information extraction and BIM information transformation.

413 BIM information extraction utilizes EXPRESS data processing techniques in a BIM information

414 extraction algorithm to extract all entities and their attributes in an IFC file into information 415 tuples based on their metadata, in a recursive and exhaustive manner. The information tuples 416 store information for each entity, the attributes of the entity, and the values of the attributes of 
417 the entity, to prepare for the following transformation process. The BIM IE algorithm 418 exhaustively extracts the values (e.g., '2O2Fr\$t4X7Zf8NOew3FNld') for each attribute (e.g., 419 global ID) of each entity (e.g., wall). Recursion is used in two ways (as illustrated in Fig. 8): (1) 420 when an entity is being extracted, not only the explicit attributes of the entity are extracted, but 421 all explicit attributes that belong to the supertype of that entity and supertype of supertype (until 422 no supertype can be found) of that entity are extracted too. For example, when a "door" entity is 423 being extracted, not only the explicit attributes “overall height” and "overall width” are extracted, 424 but all the following explicit attributes that belong to the supertypes of "door" are extracted too: 425 “global ID,” “owner history,” “name,” “description,” “object type,” “object placement,” 426 “representation,” and "tag;”and (2) if an attribute is of an aggregation data type (i.e., aggregation 427 of multiple attributes), then the member attributes of the aggregation are recursively accessed for 428 extracting their values. For example, because the attribute "related objects" of a "rel associates 429 material” is of an aggregation data type (i.e.,set data type in this case), when a "related objects" 430 instance is being processed, each of its member objectsis accessed recursively for extracting their 431 values.The late binding data access method in JSDAI is used to support the entity and attribute 432 extraction in the BIM IE algorithm. Late binding accesses each entity and attribute using 433 standard access methods in Java. 


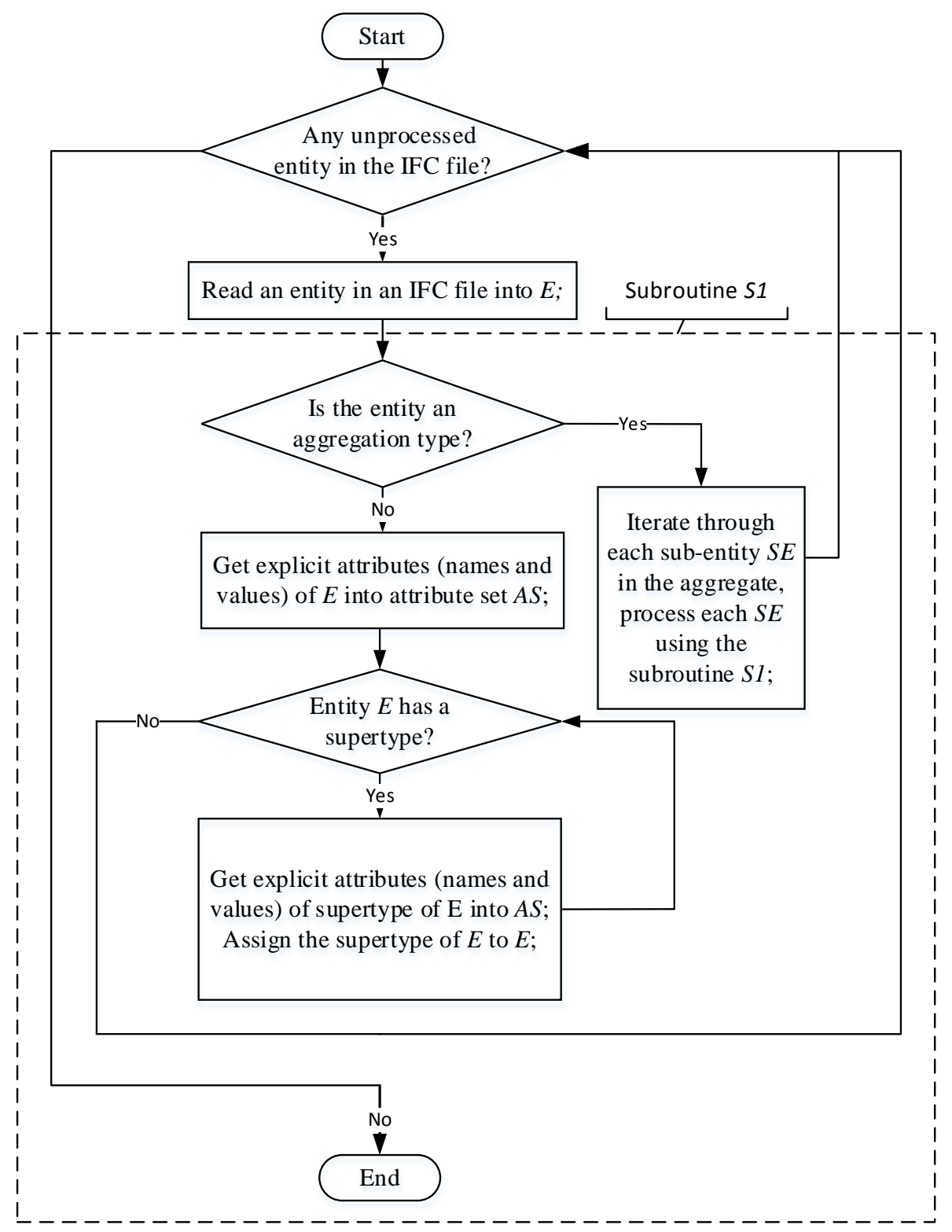

Fig.8. The BIM IE Algorithm based on two recursive processes.

BIM information transformation transforms the extracted BIM information in the information

437 tuples into logic facts (concept facts and relation facts) in two steps: initial transformation and 438 alignment transformation. Initial transformation transforms the extracted entities and their 439 attributes into concept facts and relation facts using three main initial transformation rules. These 440 rules transform elements in the entities, attributes, and values into predicate names or arguments 441 based on their metadata. For example, the first initial transformation rule in Fig. 9 converts a line 
442 in IFC data with referenced attribute values into logic facts.After initial transformation, 443 alignment transformation further transforms the generated logic facts into a logic fact 444 representation that is aligned with the predicates in the logic rules (that represent the 445 corresponding regulatory requirements). A set of semantic transformation (ST) rules are used in 446 the alignment transformation step. For example, Fig. 9 shows a set of logic facts after 447 initialtransformation and after alignment transformation using two ST rules. Compared to the 448 logic facts before alignment transformation, the logic facts after alignment transformation are more easily understandable and aligned with the logic rules.

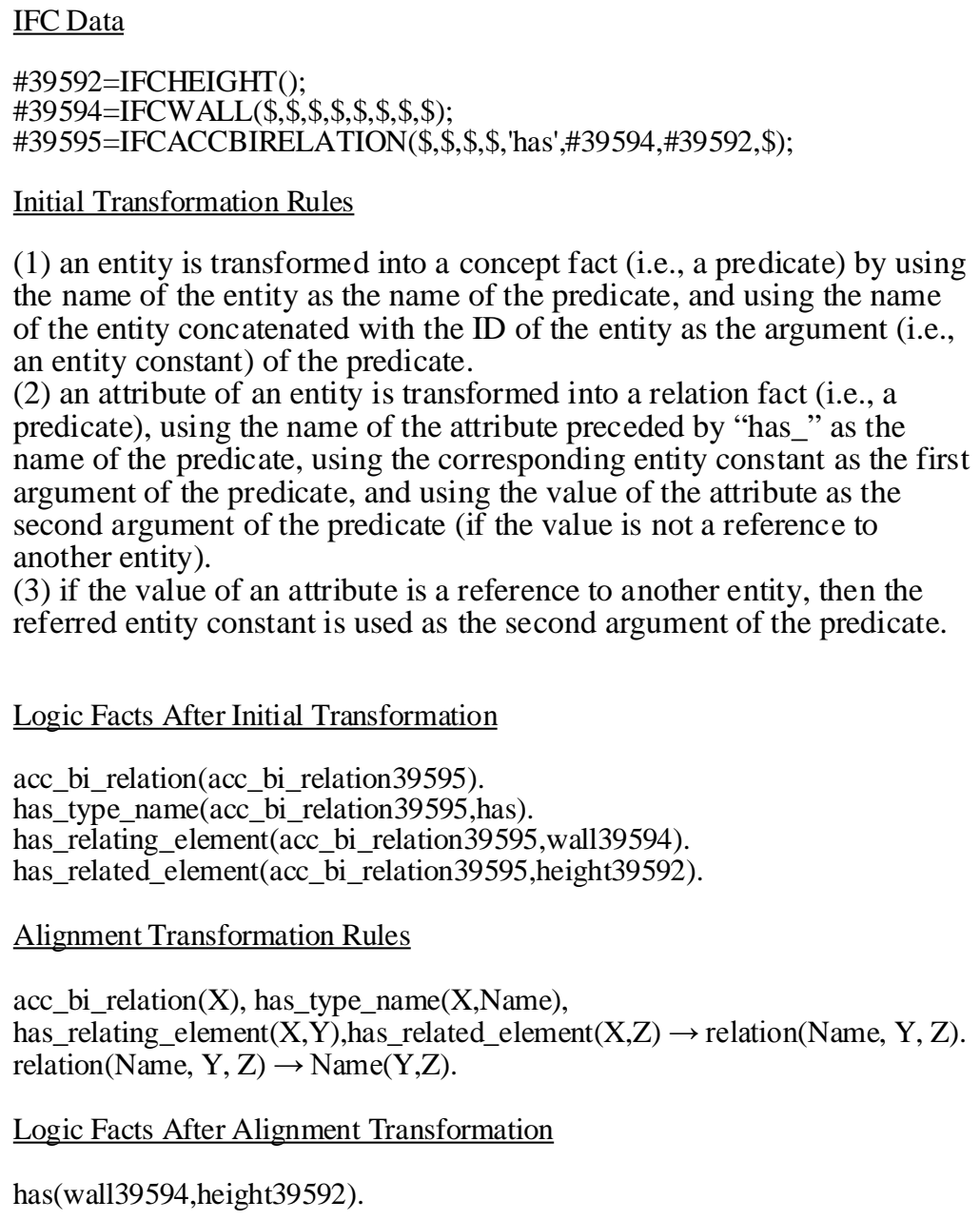

Fig.9.An example to illustrate BIM information extraction and transformation. 


\subsection{Compliance reasoning module}

453 The compliance reasoning module utilizes B-Prolog's reasoner to reason about the logic rules

454 and the logic facts and generate compliance checking reports. A set of functional built-in logic

455 clauses were developed and embedded into the system to provide basic arithmetic functions (e.g.,

456 unit conversion) and definethe sequence of execution/checking. For execution, the user specifies

457 the list of subjects (e.g., walls and doors) or subjects and attributes (e.g., walls and their heights)

458 to check, and accordingly the subjects in the specified list are sequentially checked one by one.

459 By default, a “select all” option is used.

\section{System implementation}

461 The proposed SNACC system was implemented in a proof-of-concept prototype. The main 462 platform of the prototype was built using Java programming language (Java Standard Edition 463 Development Kit 6u45). The regulatory information extraction algorithm was implemented using 464 GATE’s Processing Resources and Java programs. The following Processing Resources were 465 used: (1) the English Tokenizer, Sentence Splitter, POS Tagger, and Gazetteer in the A Nearly466 New Information Extraction (ANNIE) system for tokenization, sentence splitting, POS tagging, 467 and gazetteer compiling, (2) the Morphological Analyzer for morphological analysis, (3) the 468 Flexible Gazetteer for generating semantic features based on the ontology, and (4) the Java 469 Annotation Patterns Engine (JAPE) rules for encoding the IE rules. The information extraction 470 algorithm interacts with the Processing Resources using GATE’s API 7.0.

471 The regulatory information transformation algorithm was implemented using Python 472 programming language (Python 2.7.3). The SM rules and CR rules were coded as Python 473 conditional statements. The "re" module (i.e., regular expression module) in Python was used for 474 both extracting the syntactic and semantic features from the information tuples and conducting 
475 pattern matching. The information transformation algorithm interacts with the other modules of 476 the SNACC system (in Java) through Jython 2.2.1.

477 The BIM information extraction and transformation algorithmswere implemented in Java 478 programs and B-Prolog rules, respectively. The JSDAI runtime (JSDAI 4.3.0) was used to access 479 the information in IFC-based BIMs (IFC files) for entity and attribute extraction. String 480 processing methods in Java were used for initial transformation. Static rules and dynamic rules in 481 B-Prolog were used for alignment transformation. Static rules are rules that only use static 482 predicates. Dynamic rules are rules that use at least one dynamic predicate. A static predicate is a 483 predicate that cannot be updated during execution whereas a dynamic predicate is a predicate 484 that can be updated during execution. The rules for entity extraction, attribute extraction, and 485 initial transformation were coded as Java conditional statements. The rules for alignment 486 transformation (i.e., ST rules) were coded as B-Prolog rules.

487 The logic-based automated reasoning algorithm was implemented in Java. The functional built-in 488 logic clauses were encoded in B-Prolog. The automated reasoning algorithm interacts with the 489 logic clauses and logic reasoner through B-Prolog's bi-directional interface 7.8 with Java 490 programming language.

491 The graphical user interface of the SNACC system is shown in Fig. 10. As shown in Fig. 10, the 492 SNACC system requires the download of the GATE tool and the availability of a building 493 ontology to execute the regulatory information extraction and transformation algorithms. A user 494 could then select the regulatory document (.txt file) and the BIM (.ifc file) for automated 495 compliance checking. The information extraction and information transformation algorithms for 496 regulatory information and design information could be executed in parallel. After all 497 information have been extracted and transformed, pressing the "check compliance” button 

in Fig. 10).

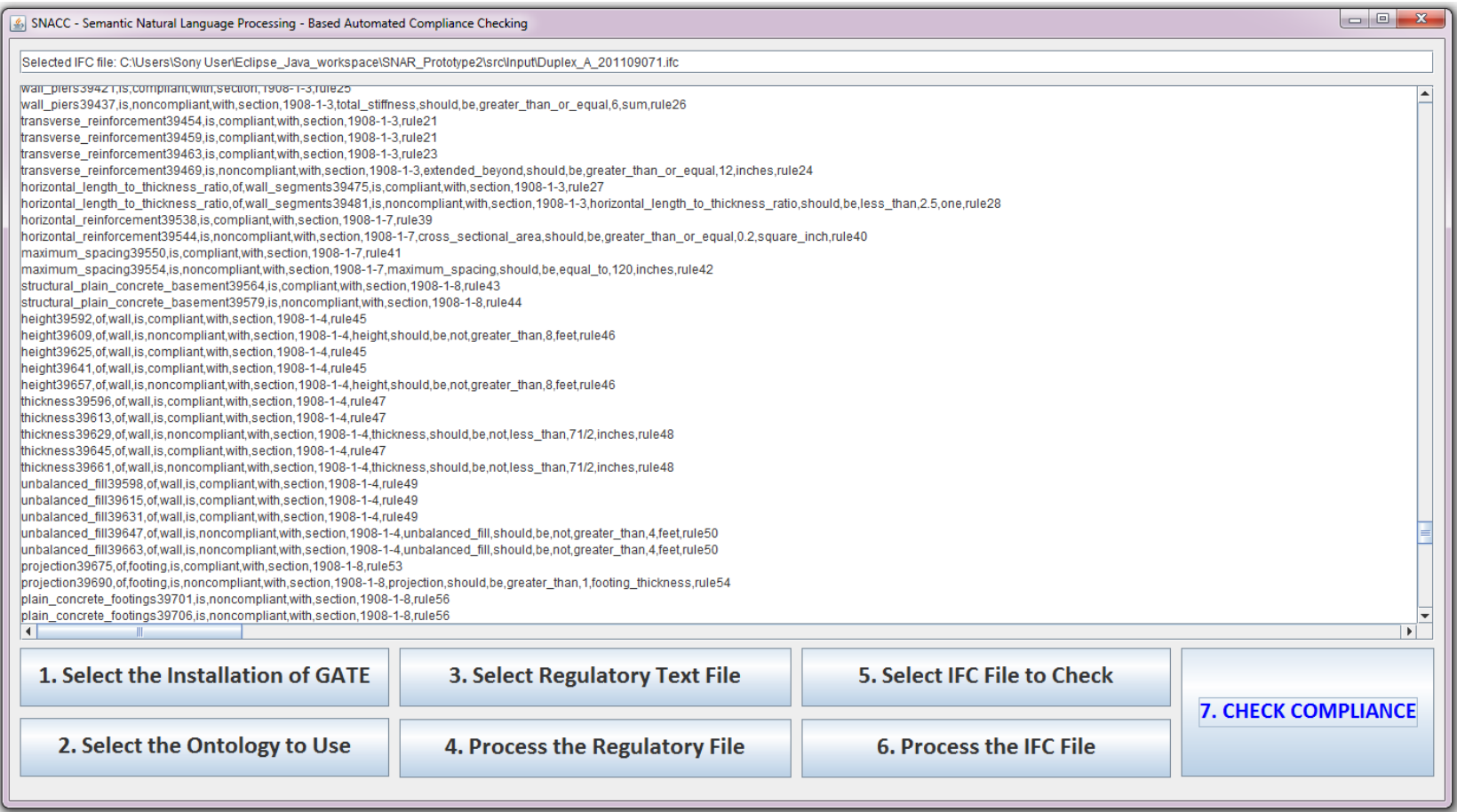

Fig. 10.Graphical user interface of the SNACC system.

\section{System testing}

The SNACC system was tested in checking the compliance of a BIM test case with Chapter 19 of IBC 2009. IBC was selected because it is predominantly adopted in the United States. Chapter 19 was then randomly selected. For the test case, it was developed based on the Duplex Apartment Project from buildingSMARTalliance of the National Institute of Building Sciences (East 2013). Design information were added in the BIM model, based on an extended version of the IFC_2X3_TC1 schema (BuildingSmart 2014)(Zhang and El-Gohary 2016a). The test case included design information for each provision in Chapter 19 of IBC 2009. The design information included both compliant and noncompliant design information. If a provision had more than one requirement, then compliant and noncompliant design information for each 
513 requirement wasincluded. For example, the following regulatory provision (RP1) is a complex

514 provision that contains three quantitative requirements: "In dwellings assigned to Seismic Design

515 Category $D$ or $E$, the height of the wall shall not exceed 8 feet (2438 $\mathrm{mm}$ ), the thickness shall not

516 be less than 71/2 inches $(190 \mathrm{~mm})$, and the wall shall retain no more than 4 feet $(1219 \mathrm{~mm})$ of

517 unbalanced fill." Thus, five information sets were created for $R P 1$ which correspond to the

518 scenarios that (1) only height is noncompliant, (2) only thickness is noncompliant, (3) only

519 unbalanced fill is noncompliant, (4) all three attributes are noncompliant, and (5) no attributes

520 are noncompliant.

\section{$521 \quad 7 \quad$ Results and discussion}

522 The ACC prototype system was evaluated using precision, recall, and F1-measure of 523 noncompliance detection. Precision is defined as the number of correctly-detected 524 noncompliance instances divided by the total number of noncompliance instances detected.

525 Recall is defined as the number of correctly-detected noncompliance instances divided by the 526 total number of noncompliance instances that should be detected. F1-measure is the harmonic 527 mean of precision and recall. A manually-developed gold standard was used for the evaluation. 528 A gold standard refers to a benchmark against which testing results are compared for evaluation.

529 The gold standard includes the ground truth of compliant and noncompliant instances.

530 The testing results are summarized in Table 2. As shown in Table 2, the recall, precision, and F1531 measure of noncompliance detection is $98.7 \%$, $87.6 \%$, and $92.8 \%$, respectively. The relevant 532 provision numbers and rule numbers for the compliant and noncompliant instances were also 533 correctly reported. For each noncompliance instance, a suggestion on how to fix the 534 noncompliance case was also correctly reported (partially shown in Fig. 10). 
Table 2.

$536 \quad$ Noncompliance detection testing results.

\begin{tabular}{|l|c|}
\hline \multicolumn{1}{|c|}{ Parameter/measure } & Result \\
\hline Number of noncompliance instances in gold standard & 79 \\
\hline Number of noncompliance instances detected & 89 \\
\hline Number of noncompliance instances correctly detected & 78 \\
\hline Recall of noncompliance detection & $98.7 \%$ \\
\hline Precision of noncompliance detection & $87.6 \%$ \\
\hline F1-measure of noncompliance detection & $92.8 \%$ \\
\hline
\end{tabular}

These high performance results show that the proposed ACC system is promising. In addition,

538 the fact that the proposed ACC system achieved higher recall (98.7\%) than precision (87.6\%)

539 shows its suitability for the ACC application; in noncompliance detection, recall is more

540 important than precision. Recall errors are more critical because they might result in missing

541 noncompliance instances, whereas precision errors could be easily double-checked and filtered

542 out by the user.

543 An error analysis was also conducted to identify the sources of the errors in noncompliance

544 detection. The noncompliance detection errors originated from errors in regulatory information

545 extraction and regulatory information transformation; there were no errors in BIM information

546 extraction, BIM information transformation, orcompliance reasoning. The errors were attributed

547 to errors made by GATE's processing resources, limitations of rules used in regulatory

548 information extraction and information transformation, and limitations of the state-of-the-art

549 NLP techniques[e.g., state-of-the-art Part-of-Speech (POS) tagging has an accuracy of around 97\%

550 (Manning 2011)]. For example, “concrete floor slab" was not successfully extracted as the

551 subject (i.e., a false negative) for the following requirementbecause of errors made by GATE's

552 processing resources: “The thickness of concrete floor slabs supported directly on the ground

553 shall not be less than 31/2 inches (89 mm)”(Provision 1910.1of IBC 2009). 


\section{Contribution to the body of knowledge}

555 This research contributes to the body of knowledge in three main ways. First, this research offers

556 a novel system for fully-automated checking of building information models for compliance with

557 building codes. The proposed system goes beyond the current state-of-the-art of ACC by

558 allowing fully-automated (1) extraction of bothregulatory and design information from

559 regulatory documents and IFC-based BIM models, respectively, and (2) alignment of the

560 representations of these two sets of information, so that they can be interpreted together in one

561 system. Second, this research offers integrated NLP and first order logic methodsfor

562 automatically extracting regulatory information from regulatory documents andautomatically

563 representing the extracted information in an ACC first order logic-based representation that is

564 used in automated ACC logic reasoning. The proposed methods/algorithms offer a novel way for,

565 both, deep information extraction (i.e., full-sentence analysis to capture the entire meaning of a

566 provision) and generalized and flexible ACC representation; both - together- enable the

567 extraction and representation of information even in long and complex provisions, which is

568 important to sustain utility and robustness of ACC system performance across different types of

569 regulatory documents and different types of provisions. Third, this research offers a novel

570 combination of NLP techniques with bothsemantic analysis and logic-based reasoning into one

571 computational framework. In this research, a set of information extraction, information

572 transformation, and automated reasoning algorithms are effectively implemented into one proof-

573 of-concept ACC system. The combined performance of all algorithms, into the system, shows

574 high automated noncompliance detection performance (98.7\%, 87.6\%, and 92.8\% recall,

575 precision, and F1-measure, respectively). 


\section{Conclusions}

577 This paper presented a unifiedsystem that integrates a set of techniques and algorithms for

578 automatically checking the compliance of BIM-based building designswith building codes. The

579 proposed system offers a fully-automated approach to ACC in construction. The approach relies

580 on the use of a set of computational techniques in an integrated manner, in one unified system.

581 The techniques include NLP, EXPRESS data processing, and logic reasoning, which are

582 collectively used for automated information extraction, automated information transformation,

583 and automated compliance reasoning. The automation is facilitated by semantic, logic-based

584 representations that are generalized and flexible.

585 The system is composed of three main modules: (1) a regulatory information extraction and

586 transformation module, which utilizes semantic natural language processing algorithms to

587 automatically extract regulatory information from building codes and transform the extracted

588 information into logic rules, (2) design information extraction and transformation module, which

589 utilizes EXPRESS data processing-based algorithms to automatically extract design information

590 from building information models and transform the extracted information into logic facts, and

591 (3) compliance reasoning module, which utilizes semantic-based logic reasoning algorithms to

592 automatically reason about the compliance of the logic facts with the logic rules. The algorithms

593 were implemented in different programming languages and integrated into one proof-of-concept

594 prototype system (the SNACC system). The integration is facilitated by the choice of a semantic

595 representation, a logic representation, a modular system architecture, and an architecture-neutral

596 platform.

597 The SNACC system was tested in checking the compliance of a BIM test case with Chapter 19 598 of IBC 2009. A recall of $98.7 \%$, a precision of $87.6 \%$, and an F1-measure of $92.8 \%$ in 
614 requires further investigation.

615 As part of their future/ongoing research work, the authors will test the proposed ACC system on 616 more building code chapters and more BIM test cases. In addition, other types of requirements 617 (e.g., existential requirements) will be tested, and different ways of handling information 618 incompleteness cases during ACC will be proposed and tested.

619 In future research - by the authors or the larger research community, the proposed information 620 extraction and transformation algorithms could also be applied to other logic-based 
621 representations such as SWRL and N3Logic. In this case the JSDAI-based BIM information

622 processing can be partially replaced by existing conversion methods such as those in Pauwels

623 and Terkaj(2016)and Beetz et al. (2009). However, in this case, further semantic transformation

624 of BIM information would still be neededto align the concept representations of the design

625 information tothose of theregulatory information.Similarly, further research could be conducted

626 to study how to best link the proposed algorithms with OWL representations and other semantic

627 modeling approaches and assess the advantages and limitations ofthe proposed methods in this

628 context. The authors expect that the proposed information extraction, information transformation,

629 and automated reasoning methods would lend themselves well to such integrative efforts.

630 However, further research is needed to study practicality, benefits, and limitations.

\section{Acknowledgements}

632 The authors would like to thank the National Science Foundation (NSF). This material is based 633 upon work supported by NSF under Grant No. 1201170. Any opinions, findings, and conclusions 634 or recommendations expressed in this material are those of the authors and do not necessarily 635 reflect the views of NSF.

\section{$636 \quad$ References}

637 AEC3. (2012). “International Code Council.”〈http://www.aec3.com/en/5/5_013_ICC.htm〉 (Jun. $638 \quad 30,2016)$.

639 Baumgärtel, K., Kadolsky, M. and Scherer, R.J. (2015).“An ontology framework for improving 640 building energy performance by utilizing energy saving regulations.”Proc., 10th European 641 Conference on Product and Process Modelling (ECPPM), ECPPM, 519-526. 
642 Beach, T.H., Kasim, T., Li, H., Nisbet, N., and Rezgui, Y. (2013).“Towards automated 643 compliance checking in the construction industry."H. Decker et al. (Eds.): DEXA 2013, Part I, LNCS 8055, 366-380.

645

646

647

648

649

650

651

652

653

654

655

656

657

658

659

660

661

662

663

664

Beach, T.H., Rezgui, Y., Li, H., and Kasim, T. (2015).“A rule-based semantic approach for automated regulatory compliance in the construction sector."Expert Systems with Applications, 42(12), 5219-5231.

Beetz, J., van Leeuwen, J., and de Vries, B. (2009). "IfcOWL: A case of transforming EXPRESS schemas into ontologies.” Artificial Intelligence for Engineering Design, Analysis and Manufacturing, 23(SP01), 89-101.

Berners-Lee, T. (2005). "Status: An early draft of a semi-formal semantics of the N3 logical properties.” <https://www.w3.org/DesignIssues/N3Logic> (Jun. 9, 2016).

Boken, P., and Callaghan, G. (2009).“Confronting the challenges of manual journal entries."Protiviti, Alexandria, VA, 1-4.

BuildingSmart.(2014). "Industry Foundation Classes (IFC) data model." <http://www.buildingsmart-tech.org/specifications/ifc-overview> (Jan 19, 2015).

Cherpas, C. (1992). "Natural language processing, pragmatics, and verbal behavior."Anal. Verbal Behav., 10, 135-147.

Choi, J., Choi, J., and Kim, I. (2014)."Development of BIM-based evacuation regulation checking system for high-rise and complex buildings.”Autom. Constr., 46, 38-49.

City of Philadelphia. (2015). "Licenses and inspections: building permits." $<$ https://business.phila.gov/licenses-and-inspections-building-permits/> (Sept. 4, 2015).

Cunningham, H., et al. (2012). “Developing language processing components with gate version 7 (a user guide).” Univ. of Sheffield, Dept. of Computer Science, Sheffield, U.K. 
665

666

667

668

669

670

671

672

673

674

675

676

677

678

679

680

681

682

683

684

685

686

Delis, E.A., and Delis, A. (1995) "Automatic fire-code checking using expert-system technology.”J. Comput. Civ. Eng., 9(2), 141-156.

Department of Buildings. (2015). "Hearing on the fiscal 2016 preliminary budget and the fiscal 2015

preliminary

mayor's

management

report.” <ttp://council.nyc.gov/html/budget/2016/Pre/dob.pdf> (Sept. 4, 2015).

Dimyadi, J., and Amor, R. (2013). “Automated building code compliance checking - where is it at?” Proc. 19th Int. CIB World Build. Congress, Brisbane, Australia.

Dimyadi, J., Clifton, C., Spearpoint, M., and Amor, R. (2014). “Regulatory knowledge encoding guidelinens for automated compliance audit of building engineering design.” Comput. Civ. Build. Eng. (2014), ASCE, Reston, VA, 536-543.

Dimyadi, J., Pauwels, P., Spearpoint., M., Clifton, C., and Amor, R.W. (2015). “Querying a regulatory model for compliant building design audit.”Proc., CIB W78 2015, Conseil International du Bâtiment (CIB), Rotterdam, The Netherlands, 139-148.

East, E.W. (2013). “Common building information model files and tools.” $<$ http://www.nibs.org/?page=bsa_commonbimfiles\&hhSearchTerms=\%22common+a nd+BIM+and+file\%22> (Jun. 27, 2014).

Eastman, C., Lee, J., Jeong, Y., and Lee, J. (2009). “Automatic rule-based checking of building designs.” Autom. Constr., 18(8), 1011-1033.

Fiatech Regulatory Streamlining Committee, (2012).“AutoCodes project: phase 1, proof-ofconcept:

final report.”<http://www.fiatech.org/images/stories/techprojects/project_deliverables/Updated_p roject_deliverables/AutoCodesPOCFINALREPORT.pdf> (Dec. 24, 2013). 
687

688

689

690

691

692

693

694

695

696

697

698

699

700

701

702

703

704

705

706

707

708

709

Fiatech. (2014). "Automated code plan checking tool-proof-of-concept (phase 2).” <http://www.fiatech.org/images/stories/projects/FiatechAutoCodesPh2-Report-Sept2015.pdf> (Jun. 16, 2016).

Garrett, J.H.Jr., and Palmer, M.E. (2014).“Delivering the infrastructure for digital building regulations.”J. Comput. Civ. Eng., 2014(28), 167-169.

Hjelseth, E. and Nisbet, N. (2011). "Capturing normative constraints by use of the semantic mark-up RASE methodology.” Proc., CIB W78 2011, Conseil International du Bâtiment (CIB), Rotterdam, The Netherlands.

Hodges, W. (2001).“Classical logic I - first-order logic.”Goble, 2001, 9-32. <http://citeseerx.ist.psu.edu/viewdoc/download?doi=10.1.1.137.4783\&rep=rep1\&type=pdf> (Dec. 26, 2013).

Horrocks, I., Patel-Schneider, P.F., Boley, H., Tabet, S., Grosof, B., and Dean, M. (2004). “SWRL: A Semantic Web Rule Language Combining OWL and RuleML.”

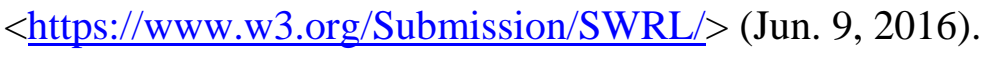

International Code Council (ICC).(2006). “2006 international building code.”2006 Int. Codes, < http://publicecodes.cyberregs.com/icod/ibc/2006f2/> (Oct. 25, 2015).

ISO. (2004). “ISO 10303-11:2004 - Part 11: Description methods: The EXPRESS language reference manual.”

$05<$ http://www.iso.org/iso/iso_catalogue/catalogue_tc/catalogue_detail.htm?csnumber=38047> (Dec. 05, 2014).

07 Java Standard Edition Development Kit 6u45.[Computer Software].Redwood Shores, CA, $08 \quad$ Oracle.

JSDAI 4.3.0.[Computer software].Kuenzell, Germany, LKSoftWare GmbH. 
710

711

712

713

714

715

716

717

718

719

720

721

722

723

724

725

726

727

728

729

730

731

Jython 2.2.1.[Computer software].<http://www.jython.org/> (May 02, 2015).

Kasim, T., Li, H., Rezgui, Y., and Beach, T. (2013). “Automated sustainability compliance checking process: proof of concept.” Proc., 13th Int. Conf. Constr. App. Vir.Real., Teesside University, Tees Valley, UK, 11-21.

Lau, G. T., and Law, K. (2004).“An information infrastructure for comparing accessibility regulations and related information from multiple sources."Proc., 10th Int. Conf. on Computational Civil and Building Engineering (ICCCBE), ISCCBE, Hong Kong, China.

$\begin{array}{lllll}\text { Los } & \text { Angeles } & \text { Times.(2015). } & \text { "Public }\end{array}$ notices.” <http://classifieds.latimes.com/classifieds?category=public_notice> (Sept. 4, 2015).

Manning, C.D. (2011). "Part-of-Speech tagging from 97\% to 100\%: is it time for some linguistics?” Proc.,12th International Conference on Intelligent Text Processing and Computational Linguistics, CICLing, Mexico.

Nguyen, T., and Kim, J. (2011)."Building code compliance checking using BIM technology."Proc., 2011 Winter Simulation Conference, Association for Computing Machinery, New York, 3400 - 3405.

Oracle.(1999). "Essentials of the Java programming language, Part 1."<http://www.oracle.com/technetwork/java/index-138747.html> (Jun. 25, 2015).

Pauwels, P., and Terkaj, W. (2016). "EXPRESS to OWL for construction industry: Towards a recommendable and usable ifcOWL ontology.” Autom. Constr., 63(Mar. 2016), 100-133.

Pauwels, P., and Zhang, S. (2015). "Semantic rule-checking for regulation compliance checking: an overview of strategies and approaches.” Proc., CIB W78 2015,Conseil International du Bâtiment (CIB), Rotterdam, The Netherlands, 619-628. 
732 Pauwels, P., Van Deursenc, D., Verstraetena, R., De Rooc, J., De Meyera, R., Van de Wallec, R., 733 Van Campenhoutb, J. (2011). “A semantic rule checking environment for building 734 performance checking.”Autom. Constr., 20(5), 506-518.

735 Portoraro, F. (2011).“Automated reasoning.”The Stanford encyclopedia of philosophy (Summer 736 2011 Edition), Edward N. Zalta <http://plato.stanford.edu/archives/sum2011/entries/reasoning-automated/> (Dec. 26, 2014).

740

741

742

743

744

745

746

747

748

749

750

751

752

753

Python v2.7.3 [Computer software].Beaverton, OR, Python Software Foundation.

Qi, J., Issa, R., Hinze, J., and Olbina, S. (2011). “Integration of safety in design through the use of building information modeling." Int. Workshop on Computing in Civil Engineering 2011, ASCE, Reston, VA, 698-705.

Saint-Dizier, P. (1994). “Advanced logic programming for language processing.”Academic Press, San Diego, CA.

Solihin, W., and Eastman, C. (2015). “A knowledge representation approach to capturing BIM based rule checking requirements using conceptual graph.” Proc., CIB W78 2015, Conseil International du Bâtiment (CIB), Rotterdam, The Netherlands, 686-695.

$\begin{array}{lllll}\text { State } & \text { of } & \text { New } & \text { Jersey.(2014). } & \text { review }\end{array}$ instructions.” <http://www.state.nj.us/dca/divisions/codes/forms/pdf_bcpr/pr_app_guide.pdf> (Sept. 4, 2015).

Tan, X., Hammad, A., and Fazio, P. (2010).“Automated code compliance checking for building envelope design.”J. Comput. Civ. Eng., 10.1061/ 1195 (ASCE)0887-3801(2010)24:2(203), 203-211. 
754 Yurchyshyna, A., Faron-Zucker, C., Thanh, N.L., and Zarli, A. (2010). "Adaptation of the

755

756

757

758

759

760

761

762

763

764

765

766

767

768

769

770

771

772

773

774

775 domain ontology for different user profiles: application to conformity checking in construction.” Web Information Systems and Technologies, Lecture Notes in Business Information Processing, 45, 128-141.

Yurchyshyna, A., Faron-Zucker, C., Thanh, N.L., and Zarli, A. (2008).“Towards an ontologyenabled approach for modeling the process of conformity checking in construction."Proc., CAiSE'08 Forum 20th Intl. Conf. Adv. Info.Sys.Eng., dblp team, Germany, 21-24.

Zhang, J., and El-Gohary, N. (2013)."Semantic NLP-based information extraction from construction regulatory documents for automated compliance checking.”J. Comput. Civ. Eng., 10.1061/(ASCE)CP.1943-5487.0000346, 04015014.

Zhang, J., and El-Gohary, N.M. (2016a).“Extending building information models semiautomatically using natural language processing techniques.”. Comput. in Civ. Eng., 10.1061/(ASCE)CP.1943-5487.0000536, C4016004.

Zhang, J., and El-Gohary, N.M. (2016b). "Semantic-based logic representation and reasoning for automated regulatory compliance checking." J. Comput. in Civ. Eng., 10.1061/(ASCE)CP.1943-5487.0000583, 04016037.Zhang, S., Teizer, J., Lee, J., Eastman, C.M., and Venugopal, M. (2013)."Building information modeling (BIM) and safety: automatic safety checking of construction models and schedules.”Autom. Constr., 29(2013), 183-195.

Zhong, B., Ding, L., Luo, H., Zhou, Y., Hu, Y., and Hu, H. (2012).“Ontology-based semantic modeling of regulation constraint for automated construction quality compliance checking.”Autom. Constr., 28, 58-70. 
776 Zhou, N. (2012). "B-Prolog user's manual (version 7.8): Prolog, agent, and constraint programming.” Afany Software. <http://www.probp.com/manual/manual.html> (Dec. 28, 2013).

779 Zouaq, A. (2011). “An overview of shallow and deep natural language processing for ontology $780 \quad$ learning."Ontology learning and knowledge discovery using the web: Challenges and recent 781 advances, IGI Global, Hershey, PA, 16-38. 